\title{
Influence of the surface band structure on electron emission spectra from metal surfaces
}

\author{
C. D. Archubi, ${ }^{1}$ M. N. Faraggi, ${ }^{1}$ V. M. Silkin, ${ }^{2,3,4}$ and M. S. Gravielle ${ }^{1}$ \\ ${ }^{1}$ Instituto de Astronomía y Física del Espacio (CONICET-UBA), \\ casilla de correo 67, sucursal 28, C1428EGA, Buenos Aires, Argentina \\ ${ }^{2}$ Donostia International Physics Center (DIPC), 20018 San Sebastián, Spain \\ ${ }^{3}$ Depto. de Física de Materiales, Facultad de Ciencias Químicas, \\ Universidad del País Vasco, Apdo. 1072, 20080 San Sebastián, Spain \\ ${ }^{4}$ IKERBASQUE, Basque Foundation for Science, 48011 Bilbao, Spain
}

(Dated: November 22, 2013)

\begin{abstract}
Electron distributions produced by grazing impact of fast protons on $\mathrm{Mg}(0001), \mathrm{Cu}(111), \mathrm{Ag}(111)$ and $\mathrm{Au}(111)$ surfaces are investigated, focusing on the effects of the electronic band structure. The process is described within the Band-Structure-Based approximation, which is a perturbative method that includes an accurate representation of the electron-surface interaction, incorporating information of the electronic band structure of the solid. For all the studied surfaces, the presence of partially occupied surface electronic states produces noticeable structures in double differential energy- and angle- resolved - electron emission probabilities from the valence band. These structures remain visible in electron emission spectra after adding contributions coming from core electrons, which might make it possible their experimental detection.

PACS numbers: $34.35 .+\mathrm{a}, 73.20 . \mathrm{At}$
\end{abstract}

\section{INTRODUCTION}

When a charged particle moves parallel to a metal surface different processes may take place [1, 2] like energy loss of the ion, charge transfer between particle and metal, inner-shell and valence-band electronic excitations and secondary electron emission. In particular, the interaction of the projectile with valence electrons of the solid produces a rearrangement of the charges inside the material, a dynamic process that gives rise to one-particle electronic transitions (electron-hole excitations) and collective excitation (plasmon) processes. For decades these processes were studied using simple representations of the unperturbed electronic states of the surface, while the emphasis was set on the description of the response of the many-electron system to the external perturbation. However, recent experimental and theoretical works showed that the band structure of different metal surfaces plays an important role in projectile induced processes 3, 5, 18. In surfaces like $\mathrm{Cu}(111), \mathrm{Ag}(111)$ and $\mathrm{Au}(111)$ it was found that the energy lost by charged particles moving at long distances from the surface is dominated by electron-hole excitations involving partially occupied surface states [12]. Also in the case of $\mathrm{Mg}(0001)$ the finite width of the surface plasmon modifies the behavior of the energy loss at long distances from the surface, being relevant for the study of the interaction mechanisms between charged particles and the internal walls of microcapilars [14, 19].

In the above mentioned articles, the influence of the surface band structure on energy loss processes was investigated making use of the dielectric formalism. This formalism involves a quantum calculation of the surface response function but describes the stopping of the incident particle by means of classical electromagnetism laws. Here we are interested in studying individual electronic transitions induced by the projectile. In particular, the work focuses on angle- and energy- resolved electron emission distributions produced by grazing impact of swift protons on metal surfaces. Such spectra are expected to provide detailed information on the electronic characteristics of the target surface.

To describe the electron emission process from the valence band we employ the Band-Structure-Based (BSB) approximation [20, 21], which is derived within the framework of the binary collisional formalism by including a precise representation of the surface interaction. For every individual electronic excitation, the BSB transition matrix is evaluated making use of the electronic states corresponding to the model potential of Ref. 22], which incorporates effects of the band structure of the metal. This potential has been successfully used in several areas 11 14, 20 24], reproducing properly the projected energy gap and the energies of the surface and first image states. Within the BSB method, the dynamic response of the medium to the incident charge is obtained from the unperturbed electronic wave functions by using the linear response theory.

In a previous work 21] the BSB approximation was applied to study electron emission induced by grazing incidence of fast protons on a $\mathrm{Be}(0001)$ surface. It was found that the distribution of ejected electrons presents prominent signs of the surface band structure, with pronounced shoulders due to the contribution of partially occupied surface electronic states (SESs). In this paper the research is extended to consider electron emission from $\mathrm{Cu}(111), \mathrm{Ag}(111), \mathrm{Au}(111)$ and $\mathrm{Mg}(0001)$ surfaces, for which it is foreseeable that band structure effects leave footprints on the electron emission spectra like the ones observed in stopping processes. Contribu- 
tions from the inner shells of surface atoms, calculated with the Continnum-distorted-wave-Eikonal-initial-state (CDW-EIS) approximation, are also included in the spectra in order to determine the energy and angular range where band structure effects might be experimentally detected. In addition, with the aim of investigating the dependence on the incidence conditions, the influence of the projectile trajectory is analyzed.

The paper is organized as follows. In Sec. II we summarize the theoretical model, results are presented and discussed in Sec. III, and Sec. IV contains our conclusions. Atomic units are used unless otherwise stated.

\section{THEORETICAL METHOD}

We consider a projectile $P$ that impinges grazingly on a metal surface, inducing the one-electron transition $i \rightarrow f$, where the state $i$ belongs to the valence band while the state $f$ lays in the continuum. Within the binary collisional formalism, the corresponding transition probability per unit path reads [25]:

$$
P_{i f}(Z)=\frac{2 \pi}{v_{s}} \delta(\Delta)\left|T_{i f}\right|^{2}
$$

where $Z$ is the projectile distance to the surface, $v_{s}$ is the component of the projectile velocity parallel to the surface plane, and the Dirac delta function $\delta(\Delta)$ expresses the energy conservation, with

$$
\Delta=\vec{v}_{s} \cdot\left(\vec{k}_{f s}-\vec{k}_{i s}\right)-\left(E_{f}-E_{i}\right)
$$

$\vec{k}_{i s}\left(\vec{k}_{f s}\right)$ the initial (final) electron momentum parallel to the surface, and $E_{i}\left(E_{f}\right)$ the initial (final) electron energy. In Eq. (11) $T_{\text {if }}$ represents the T-matrix element, which is evaluated within a first-order perturbation theory as:

$$
T_{i f}=\left\langle\Phi_{f}|V| \Phi_{i}\right\rangle
$$

where $\Phi_{i}\left(\Phi_{f}\right)$ is the initial (final) unperturbed electronic state, evaluated with the BSB model, and $V$ denotes the perturbative potential produced by the external charge.

By assuming translational invariance in the plane parallel to the surface, the BSB unperturbed states, $\Phi_{i}=$ $\Phi_{\vec{k}_{i s}, n_{i}}$ and $\Phi_{f}=\Phi_{\vec{k}_{f s}, n_{f}}$, are expressed as:

$$
\Phi_{\vec{k}_{s}, n}(\vec{r})=\frac{1}{2 \pi} \exp \left(i \vec{k}_{s} \cdot \vec{r}_{s}\right) \phi_{n}(z)
$$

where $\vec{r}=\left(\vec{r}_{s}, z\right)$ is the position vector of the active electron, with $\vec{r}_{s}$ and $z$ being the components of $\vec{r}$ parallel and perpendicular, respectively, to the surface plane. The function $\phi_{n}(z)$ represents the eigenfunction of the one-dimensional Schrödinger equation associated with the surface potential of Ref. [22] with eigenenergy $\varepsilon_{n}$. By using slab geometry, the eigenfunctions $\phi_{n}(z)$ can be classified as symmetric (S) or antisymmetric (A) according to the reflection symmetry properties with respect to the center of the slab. They read:

$$
\begin{gathered}
\phi_{n}^{(S)}(z)=\frac{1}{\sqrt{L}} c_{n}^{(S)}(0)+\frac{1}{\sqrt{L}} \sum_{l=1}^{N} c_{n}^{(S)}(l) \cos \left(\frac{2 \pi l}{L} \widetilde{z}\right) \\
\phi_{n}^{(A)}(z)=\frac{1}{\sqrt{L}} \sum_{l=1}^{N} c_{n}^{(A)}(l) \sin \left(\frac{2 \pi l}{L} \widetilde{z}\right)
\end{gathered}
$$

where $L$ is the length of the unit cell, $2 N+1$ is the number of basis functions, and the coefficients $c_{n}^{(j)}(l)$, $j=S, A$, are obtained numerically [12]. The coordinate $\widetilde{z}=z+d_{s}$ represents the normal distance measured with respect to the center of the slab, which is placed at a distance $d_{s}$ from the surface plane.

In Eq. (3) the potential $V$ is expressed as $V=$ $V_{P e}+V_{i n d}$, where $V_{P e}=-Z_{P} / r_{P}$ denotes the Coulomb projectile potential, with $Z_{P}$ the projectile charge and $r_{P}$ the electron-projectile distance, and $V_{\text {ind }}$ represents the surface potential induced by the incident ion moving at a distance $Z$ from the surface plane. The potential $V_{\text {ind }}$ is obtained from the two-dimensional Fourier transform of the density-density response function, which is calculated within the linear response theory by employing the BSB unperturbed electronic states given by Eq. (44) [12, 26].

The differential electron transition probability from the valence band to a given final state $f$ with momentum $\vec{k}_{f}, d P_{v b} / d \vec{k}_{f}$, is derived by integrating Eq. (11) along the classical projectile path, after adding the contributions coming from the different initial states. That is,

$$
\frac{d P_{v b}}{d \vec{k}_{f}}=\int_{-\infty}^{+\infty} d X\left[\sum_{i} \rho_{e} \Theta\left(-E_{W}-E_{i}\right) P_{i f}(Z(X))\right]
$$

where $Z(X)$ is the projectile trajectory, with $X$ the coordinate along the incidence direction, $\rho_{e}=2$ takes into account the spin states, and the unitary Heaviside function $\Theta$ restricts the initial states to those contained inside the Fermi sphere, with $E_{W}$ the work function. Notice that within the BSB model the final electronic states $\Phi_{f}$ present a well defined momentum only in the direction parallel to the surface plane. Then, in order to determine $\vec{k}_{f}=\left(\vec{k}_{f s}, k_{f z}\right)$ it is necessary to define an effective electron momentum perpendicular to the surface as $k_{f z}=\sqrt{2 \varepsilon_{n_{f}}}$, where $\varepsilon_{n_{f}}$ is the eigenenergy associated with the final one-dimensional wave function $\phi_{n_{f}}(z)$. More details of the BSB approximation can be found in Ref. [20].

\section{RESULTS}

We apply the BSB approximation to investigate electron emission spectra produced by grazing scattering 
of protons from different metal surfaces - $\mathrm{Mg}(0001)$, $\mathrm{Cu}(111), \mathrm{Ag}(111)$ and $\mathrm{Au}(111)$ - considering incidence velocities in the high energy range, i.e. ranging from 1.5 a.u. to 3.5 a.u. At this impact energies electron capture processes are negligible and protons can be treated as bare ions along the whole path [27]. To evaluate the classical projectile trajectory we employed the ZieglerBiersack-Littmark potential [28] plus the BSB induced potential, which was derived in a consistent way by using the linear response theory [12].

The differential probability of electron emission from the valence band, $d P_{v b} / d \vec{k}_{f}$, was obtained from Eq. (7) by interpolating the $P_{i f}$ function, given by Eq. (1), from data corresponding to 24 different $Z$ distances. The $T_{i f}$ elements were calculated by using the BSB wave functions of Eq. (4), where the one-dimensional wave functions $\phi_{n}$ were derived by following the same procedure as in Refs. [20, 21, 23]. A slab formed by 40 atomic layers was used for the different targets, while the number of layers associated with the vacuum was chosen as 20 for $\mathrm{Cu}, \mathrm{Ag}$ and $\mathrm{Au}$, and 10 for the case of $\mathrm{Mg}$. The length of the unit cell $(L)$ considered for every material varies between $250 \AA$ and $300 \AA$ and the sums in Eqs. (5D) and (6) run up to $N=150$. The Fermi energy level was determined from the work function values reported in Ref. 222. Notice that all the studied surfaces present SESs that are partially occupied (see Fig. 1), with energies varying from $\varepsilon_{S E S}=-4.62 \mathrm{eV}$ for silver to $\varepsilon_{S E S}=-6.02 \mathrm{eV}$ for gold, the energy values being measured with respect to the vacuum level.

Within the BSB model two $\phi_{n_{f}}(z)$ functions - the symmetric one and the antisymmetric one - are associated with the same energy $\varepsilon_{n_{f}}$ in the thick slab limit. This representation does not allow to distinguish electrons emitted inside the solid from those ejected towards the vacuum semi-space. Then, as a first estimate we considered that ionized electrons emitted to the vacuum region represent approximately a $50 \%$ of the total ionized electrons from the valence band [20, 23].

With the aim of presenting an overall scenario of the influence of the electronic band structure for the different materials, in Fig. 2 we show $d P_{v b} / d \vec{k}_{f}$, as a function of the electron energy, for protons impinging on $\mathrm{Mg}, \mathrm{Cu}$, $\mathrm{Ag}$ and $\mathrm{Au}$ surfaces with the incidence velocity $v=2 \mathrm{a} . \mathrm{u}$. and the glancing angle $\alpha=0.1^{\circ}$. In the figure, results for the ejection angle $\theta_{e}=30^{\circ}$, measured with respect to the surface in the scattering plane, are displayed by using the same scale for all the cases. These spectra show the typical double-peaked structure associated with soft and binary single-particle collisions, respectively, with conduction electrons [29]. But in addition to such structures, the BSB curves exhibit a noticeable superimposed bulge in the high electron energy region, which disappears completely when partially occupied SESs are not included in the calculations. The shape and size of this elevation depends on the material, looking like a large shoulder for $\mathrm{Mg}, \mathrm{Cu}$ and $\mathrm{Au}$, while for $\mathrm{Ag}$ the structure resembles a small protuberance of the electron distribution.
We found that the contribution coming from SESs, also shown in the figure, is responsible for the superimposed structure of the electron spectrum. This is due to the fact that SESs present highly peaked electron densities near the surface, as shown in Fig. 1, which favors the electron emission when the projectile moves far from the surface plane. As a result, the SES contribution is more relevant when, in the selvage region, differences between the electronic density associated with the SES and those corresponding to other occupied electronic states are larger, as it happens for the copper, silver and gold surfaces, for which the differences rise up to a factor larger than 70. On the other hand, when the electron-surface interaction is represented by a finite step potential (jellium model) 25], without taking into account the electronic band structure, the electron emission distributions display a smooth behavior as a function of the electron energy, also observed for $\mathrm{Be}(0001)$ surfaces [21].

In order to analyze the angular dependence of the SES contribution, differential probabilities for electron emission from the valence band of $\mathrm{Cu}(111)$ are plotted in Fig. 3 , as a function of the electron energy, for emission angles $\theta_{e}$ ranging from $20^{\circ}$ to $70^{\circ}$. For the smaller $\theta_{e}$ values the SES bulge is placed around the energy of the binary maximum and when the ejection angle increases, the SES structure moves gradually to the low energy region. Simultaneously, an additional SES peak arises at low energies, which ends up joined to the SES shoulder for $\theta_{e} \succeq 60^{\circ}$. This behavior is ruled by the energy conservation imposed by the delta function of Eq. (2), which determines the maximum $\left(k_{S E S}^{(+)}\right)$and minimum $\left(k_{S E S}^{(-)}\right)$final momenta reached by transitions from occupied SESs. These momenta verify

$$
k_{S E S}^{( \pm)}=v_{s} \cos \theta_{e}+\sqrt{R_{ \pm}^{2}-v_{s}^{2} \sin ^{2} \theta_{e}}
$$

where $R_{ \pm}^{2}=\left(k_{S E S} \pm v_{s}\right)^{2}+2 \varepsilon_{S E S}$, with $k_{S E S}=$ $\sqrt{-2\left(E_{W}+\varepsilon_{S E S}\right)}$. The right hand side of Eq (8) decreases when $\theta_{e}$ increases and, consequently, the maximum and minimum energies associated with SES emission shift to lower values.

Even though for all the considered surfaces, SES structures are clearly visible in electron emission spectra from the valence band, in experimental electron distributions [30] there is another source of ejected electrons - the inner shells of surface atoms - which might hide SES effects. To evaluate the inner-shell emission yield we employ a semiclassical formalism [29] that describes the multiple collisions of the incident ion with the surface atoms as single encounters with outermost atoms along the projectile path. In the model the core emission probability per unit path is expressed in terms of atomic probabilities, which are evaluated within the CDW-EIS approximation. The CDW-EIS approach is a distorted wave method that accounts for the proper asymptotic conditions [31], including the distortion produced by the projectile in both the initial and final states. In the calculation of the atomic 
probability we have taken into consideration the full dependency of the CDW-EIS transition amplitude on the impact parameter, that is, not only on the modulus of the impact parameter but also on its direction.

Total emission probabilities obtained as the sum of valence and core contributions are plotted in Fig. 4, together with the partial valence and inner-shell distributions, for electron emission from a $\mathrm{Cu}(111)$ surface with different ejection angles. The core emission probability from the copper surface was evaluated by including the 3d- level only, since contributions coming from deeper shells are expected to be negligible. To represent the $3 \mathrm{~d}$ initial state of $\mathrm{Cu}$ we used the Hartree-Fock wave function corresponding to the $\mathrm{Cu}^{+}$ion [32], considering that the outermost electron was assigned to the valence band. The final continuum state, associated with the electron ejected from the $3 \mathrm{~d}$ - level, was described as a Coulomb wave function with an effective charge satisfying the initial binding energy. The figure shows that core electrons give rise to a probability that decreases evenly when the electron energy increases. It represents the main contribution to the electron emission spectra in almost the whole energy and angular range. However, signatures of the SES emission are still present in total electron distributions. At intermediate ejection angles, the SES structure of the valence-band distribution is also visible, albeit weakened, in the total emission spectrum, producing an increase of total probability around the SES position that varies between $20 \%$ and $35 \%$ approximately. In turn, for $\theta_{e} \succeq 60^{\circ}$ SES effects are reflected as a change in the slope of the electron energy distribution. Notice that under the condition of grazing incidence, transport effects are expected to play a minor role [33], at least for the electron ejection angles considered here, and consequently, present theoretical spectra might be directly compared with experimental data.

When the atomic number of surface atoms increases, the inner-shell contribution to the electron emission process augments, as it happens for $\mathrm{Ag}$ and $\mathrm{Au}$ surfaces, displayed in Figs. 5 and 6, respectively. For silver, innershell emission corresponding to the $4 \mathrm{~d}$ - level was calculated by using the Hartree-Fock wave function of $\mathrm{Ag}^{+}$ 32 , while for gold the core contribution from the $5 \mathrm{~d}-$ level was evaluated by employing the relativistic wave function of Ref. [34]. In both cases, at the smaller ejection angles, core emission represents the dominant mechanism that partially conceals surface band structure effects in electron emission spectra. For $\mathrm{Ag}(111)$, a small SES structure is perceivable in total electron distributions for ejection angles lower than $70^{\circ}$, while for larger angles the SES contribution produces a change of the slope at low electron energies, also observed for $\mathrm{Cu}$ surfaces. But for $\mathrm{Au}(111)$, despite the remarkable contribution of the electron emission from partially occupied SESs, at intermediate angles its effects are almost completely covered by the inner-shell contribution, being only noticeable for $\theta_{e} \succeq 70^{\circ}$.

On the contrary, for $\mathrm{Mg}(0001)$ valence-band electrons provide the main contribution to the electron emission process, as shown in Fig. 7. In this case, inner-shell emission from the L- shell of $\mathrm{Mg}$ cores 35] is more than one order of magnitude lower than the valence-band contribution, except for the lower $\theta_{e}$ values, precisely in the energy region where electron emission from the valence band is forbidden as a result of energy conservation (Eq. (2)). Then, although SES effects for $\mathrm{Mg}(0001)$ are weaker than for the previous surfaces, they are appreciable in total electron distributions for a wide range of ejection angles.

Finally, we address the study of the influence of the incidence conditions on SES effects, taking as prototype the $\mathrm{Cu}(111)$ surface. In Fig. 8.a we plot $d P_{v b} / d \vec{k}_{f}$ for protons impinging grazingly on $\mathrm{Cu}(111)$ with velocities ranging from $v=1.5$ a.u. to $v=3.5$ a.u. The ejection angle was chosen as $\theta_{e}=30^{\circ}$. We found that the SES shoulder shifts to higher electron energies as the projectile velocity increases, in accord with Eq. (8), but its relative contribution changes moderately as the velocity varies. However, when the incidence angle $\alpha$ augments keeping the velocity as a constant, the SES structure becomes smaller, producing only a smooth shoulder in the electron emission probability for $\alpha=0.75^{\circ}$, as shown in Fig. 8.b. This behavior is due to the fact that large incidence angles allow protons to reach distances closer to the surface, inducing a strong electron emission from different occupied electronic states. But when projectiles move far away from the surface plane, as it happens for the lower $\alpha$ values, only SES electrons are strongly affected by the external perturbation, giving rise to a remarkable SES contribution. Similar behavior was also observed for the other surfaces. Then, for the studied surfaces SESs might be experimentally probed by proton impact with glancing angles.

\section{CONCLUSION}

Electron emission spectra produced by grazing incidence of protons on $\mathrm{Mg}(0001), \mathrm{Ag}(111), \mathrm{Cu}(111)$, and $\mathrm{Au}(111)$ surfaces have been studied, including valenceband and inner-shell contributions. For all the considered surfaces, BSB differential emission probabilities from the valence band display noticeable structures due to the presence of partially occupied SESs. Such structures are related to the high localization of the electronic density of the SES around the selvage region, which promotes the electron emission process for projectiles moving outside the solid. Consequently, SES structures are more pronounced for glancing incidence angles, moving to higher energies as the incidence velocity increases.

We found that SES structures are clearly visible in total emission spectra for $\mathrm{Mg}, \mathrm{Cu}$ and $\mathrm{Ag}$ surfaces. But for $\mathrm{Au}$, band structure effects become softened and even completely covered by the inner-shell emission, being only detectable at large ejection angles. We hope the present work will prompt experimental research on the 
subject.

\section{Acknowledgments}

This work was partially supported by the Universidad de Buenos Aires, the Agencia Nacional de Promoción
Científica y Tecnológica of Argentina, the Consejo Nacional de Investigaciones Científicas y Técnicas (CONICET)
[1] A. Arnau et al., Surf. Sci. Rep. 27, 117 (1997).

[2] H. Winter, Phys. Rep. 367, 387 (2002).

[3] D. Goebl et al., Phys. Rev. B 84, 165428 (2011).

[4] A. Sarasola, V. M. Silkin, and A. Arnau, Phys. Rev. B 75, 045104 (2007).

[5] A. Sindona et al., Surf. Sci. 601, 1205 (2007).

[6] M. Pisarra, P. Ricardi, A. Cupolillo, A. Sindona, and L. S. Caputi, Nanosci. Nanotechnology Lett. 4, 1100 (2012).

[7] A. Sindona et al., Nucl. Instrum. Methods Phys. Res. B 269, 938 (2011).

[8] T. Hecht, H. Winter, A. G. Borisov, J. P. Gauyacq, and A. K. Kazansky, Phys. Rev. Lett. 84, 2517 (2000).

[9] T. Hecht, H. Winter, A. G. Borisov, J. P. Gauyacq, and A. K. Kazansky, Faraday Discuss. 117, 27 (2000).

[10] A. G. Borisov, A. Mertens, S. Wethekam, and H. Winter, Phys. Rev. A 68, 012901 (2003).

[11] M. Alducin, V. M. Silkin, J. I. Juaristi, and E. V. Chulkov, Phys. Rev. A 67, 032903 (2003).

[12] V. M. Silkin, J. M. Pitarke, E. V. Chulkov, and P. M. Echenique, Phys. Rev B 72, 115435 (2005).

[13] V. M. Silkin, M. Alducin, J. I. Juaristi, E. V. Chulkov, and P. M. Echenique, J. Phys. Condens. Matter 20, 304209 (2007).

[14] N. Stolterfoht et al., Phys. Rev. Lett. 88, 133201 (2002).

[15] L. Vattuone et al., Phys. Rev. Lett. 110, 127405 (2013).

[16] B. Diaconescu et al., Nature (London) 448, 57 (2007).

[17] S. J. Park and R. E. Palmer, Phys. Rev. Lett. 105, 016801 (2010).

[18] M. Jahn et al., Phys. Rev. B 86, 085453 (2012).

[19] K. Tőkési, X. M. Tong, C. Lemell, and J. Burgdörfer, Phys. Rev. A 72, 022901 (2005).

[20] M. N. Faraggi, M. S. Gravielle, and V. M. Silkin, Phys. Rev. A 69, 042901 (2004).

[21] C. D. Archubi, M. S. Gravielle, and V. M. Silkin, Phys. Rev. A 84, 012901 (2011).

[22] E. V. Chulkov, V. M. Silkin, and P. M. Echenique, Surf. Sci. 391, L1217 (1997); 437, 330 (1999).

[23] M. N. Faraggi, M. S. Gravielle, M. Alducin, J. I. Juaristi, and V. M. Silkin, Phys. Rev. A 72, 012901 (2005).

[24] C. A. Ríos Rubiano, M. S. Gravielle, D. M. Mitnik, and V. M. Silkin, Phys. Rev. A 85, 043422 (2012).

[25] M. S. Gravielle, Phys. Rev. A 58, 4622 (1998).

[26] A. G. Eguiluz, Phys. Rev. Lett. 51, 1907 (1983).

[27] M. S. Gravielle and J. E. Miraglia, Phys. Rev. A 50, 2425 (1994).

[28] J.F. Ziegler, J.P. Biersack and U. Littmark, The Stopping and Range of Ions in Solids, Vol. 1 (Pergamon Press, New York, 1985).

[29] M. S. Gravielle, Phys. Rev. A 62, 062903 (2000).

[30] O. Grizzi, E. A. Sanchez, S. Lacombe, and V. A. Esaulov, Phys. Rev. B 68, 085414 (2003).

[31] D S F Crothers and J F McCann, J. Phys. B 16, 3229 (1983).

[32] E. Clementi and C. Roetti, Atomic Data and Nuclear Data Tables 14, 177 (1974), pg. 237.

[33] K. Kimura, G. Andou, and K. Nakajima, Phys. Rev. Lett. 81, 5438 (1998).

[34] C. C. Montanari, D. M. Mitnik, C. D. Archubi, and J. E. Miraglia, Phys. Rev. A 80, 012901 (2009).

[35] The corresponding initial states were described by using Hartree-Fock wave functions for $\mathrm{Mg}^{++}$, extracted from Ref. [32], pg. 332.

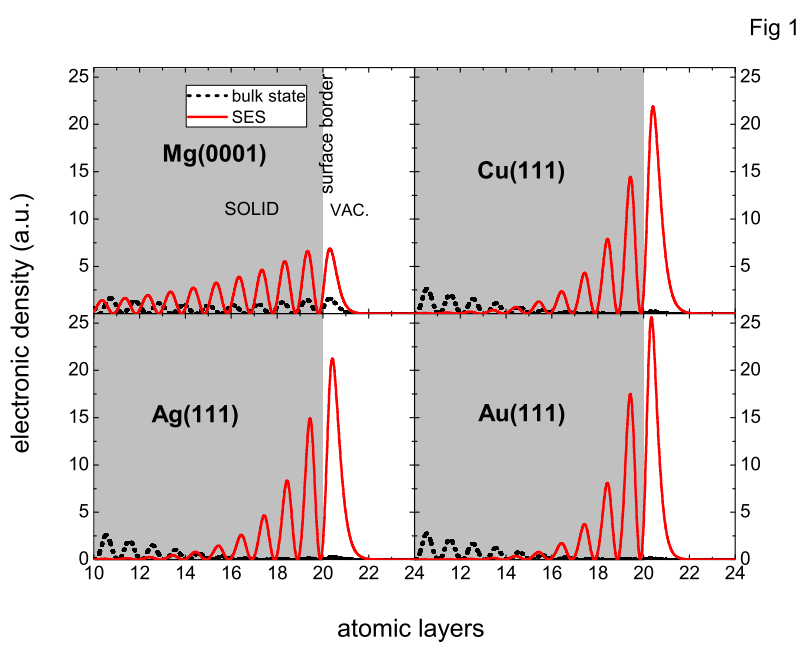

FIG. 1: (Color online) Comparison between the electronic density of the SES and the one corresponding to a bulk-like state with a close energy value for a) $\operatorname{Mg}(0001)$, b) $\mathrm{Cu}(111)$, c) $\operatorname{Ag}(111)$, and d) $\mathrm{Au}(111)$. 
Fig. 2

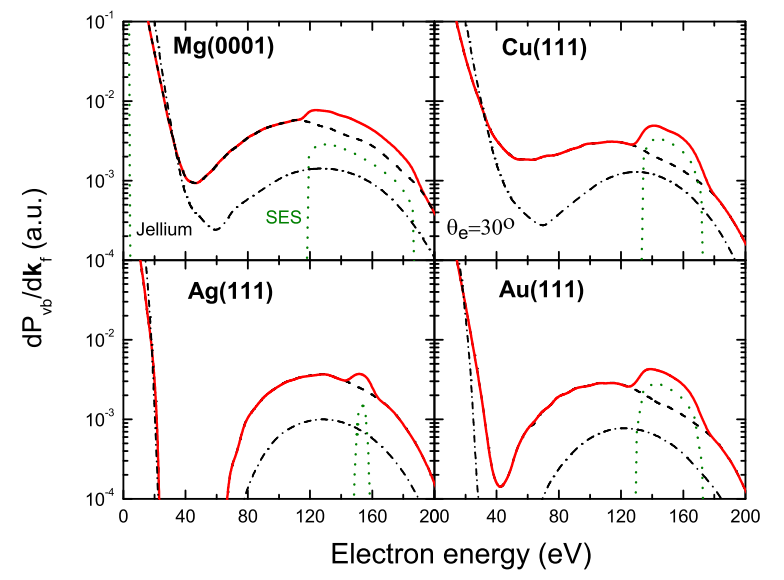

FIG. 2: (Color online) Differential probability of electron emission from the valence band, as a function of the electron energy, for $100 \mathrm{keV}$ protons impinging with the glancing angle $\alpha=0.1^{\circ}$ on a) $\operatorname{Mg}(0001)$, b) $\mathrm{Cu}(111)$, c) $\mathrm{Ag}(111)$, and d) $\mathrm{Au}(111)$. The electron ejection angle is: $\theta_{e}=30^{\circ}$, measured with respect to the surface in the scattering plane. Solid red line, BSB results including SES contribution; dashed black line, BSB results without the SES contribution; dotted green line, SES contribution; dash-dotted black line, results obtained within the jellium model [25]. 
Surface: Cu (111)

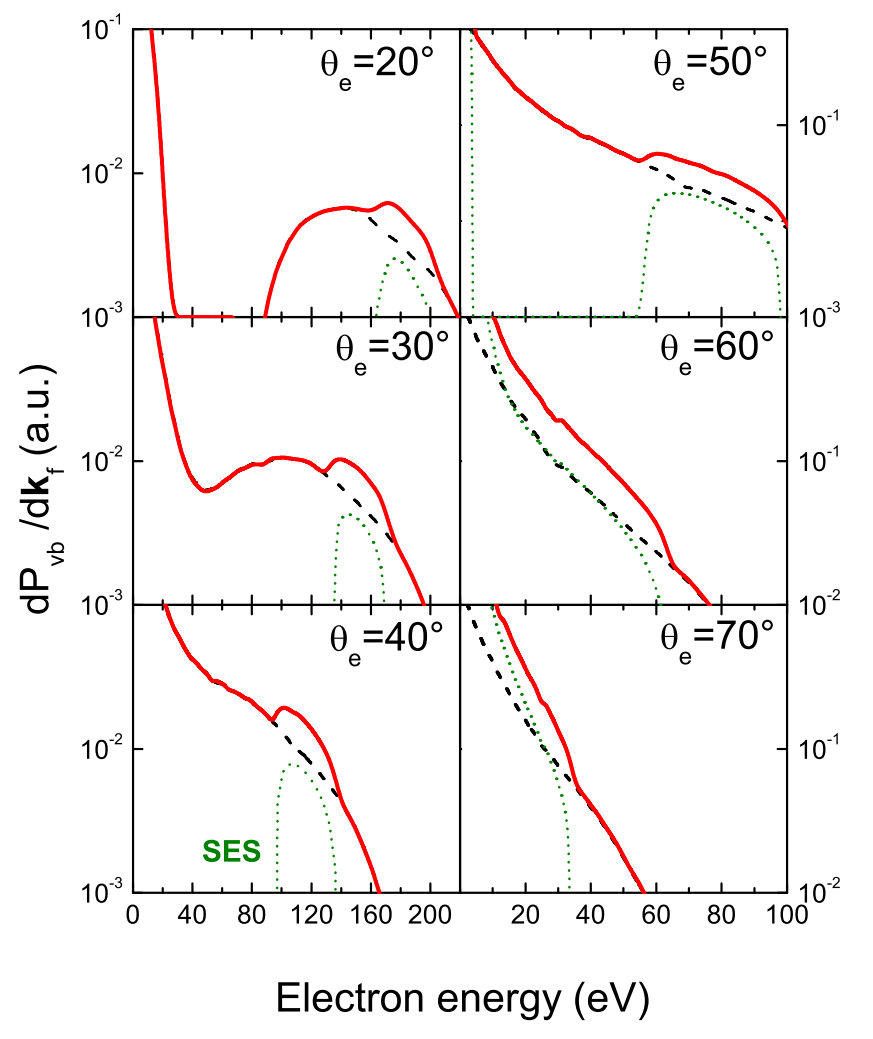

FIG. 3: (Color online) Influence of the SES in differential electron emission probabilities for $100 \mathrm{keV}$ protons impinging on a $\mathrm{Cu}(111)$ surface with an incidence angle $\alpha=0.5^{\circ}$. The electron ejection angles are $\theta_{e}=20^{\circ}, 30^{\circ}, 40^{\circ}, 50^{\circ}, 60^{\circ}$ and $70^{\circ}$, respectively, all of them measured with respect to the surface in the scattering plane. Solid red line, BSB electron emission spectrum; dashed black line, BSB electron emission spectrum without including the SES contribution; dotted green line, SES contribution. 


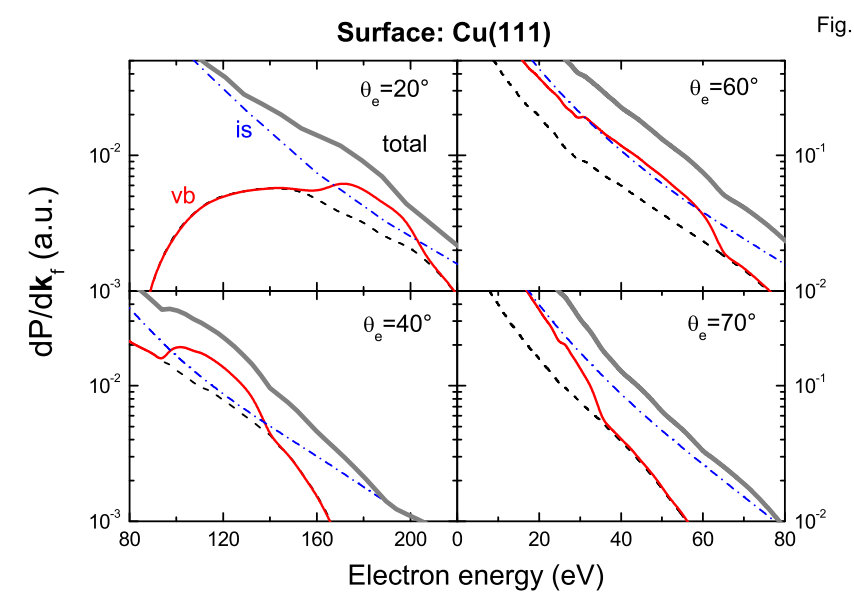

FIG. 4: (Color online) Similar to Fig. 3 for total emission probability, including valence-band and inner-shell contributions. The ejection angles are $\theta_{e}=20^{\circ}, 40^{\circ}, 60^{\circ}$, and $70^{\circ}$, respectively. Solid thick gray line, total emission probability obtained by adding valence band and core contributions, as explained in the text; solid thin red line, BSB valence emission probability; dashed black line, BSB probability without including the SES contribution; dot-dashed blue line, inner-shell emission probability.

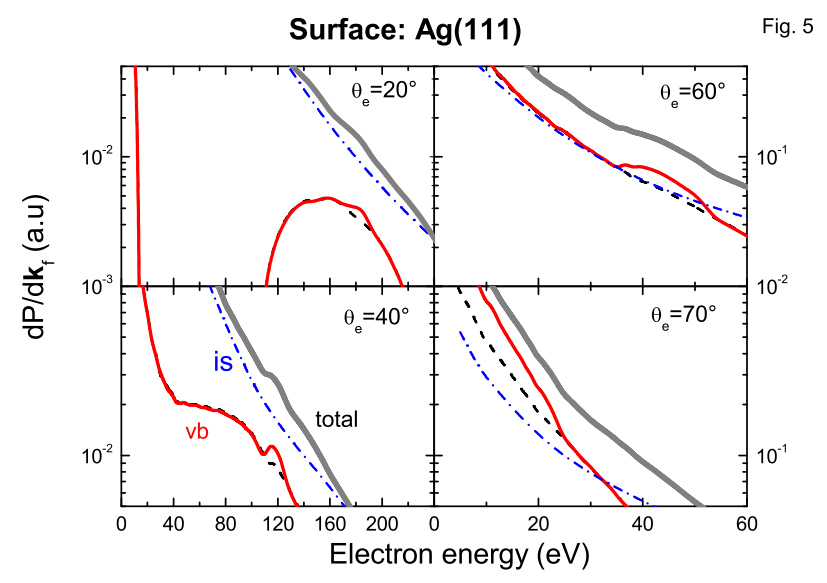

FIG. 5: (Color online) Similar to Fig 4 for a $\mathrm{Ag}(111)$ surface. 


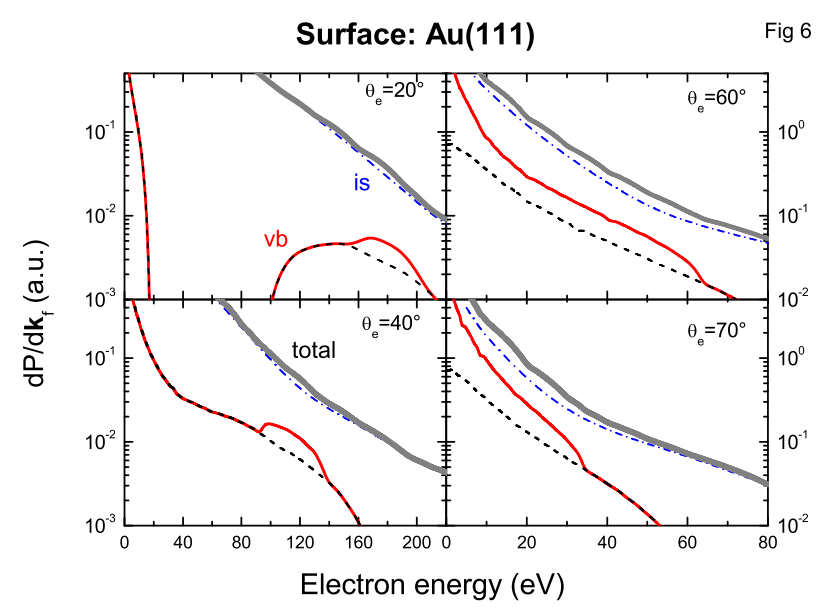

FIG. 6: (Color online) Similar to Fig 4 for a $\mathrm{Au}(111)$ surface.

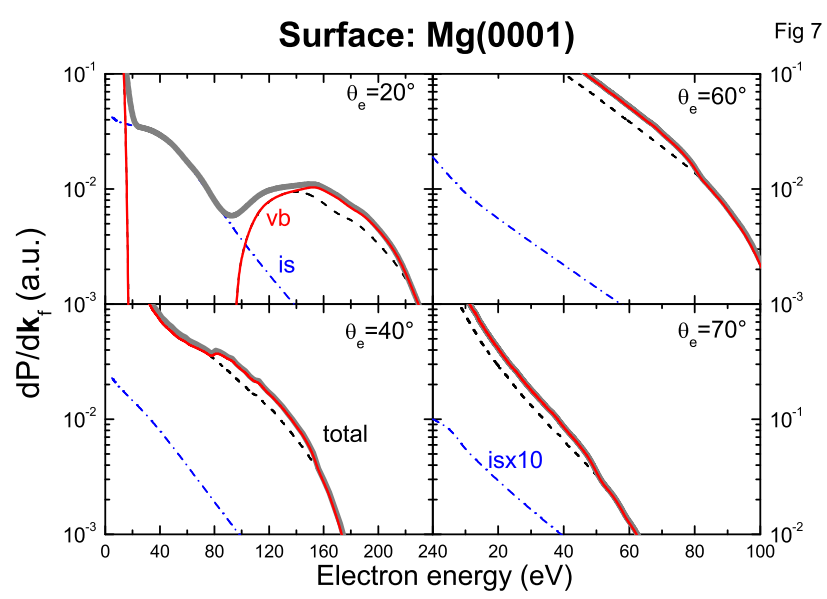

FIG. 7: (Color online) Similar to Fig 4 for a $\mathrm{Mg}(0001)$ surface. 


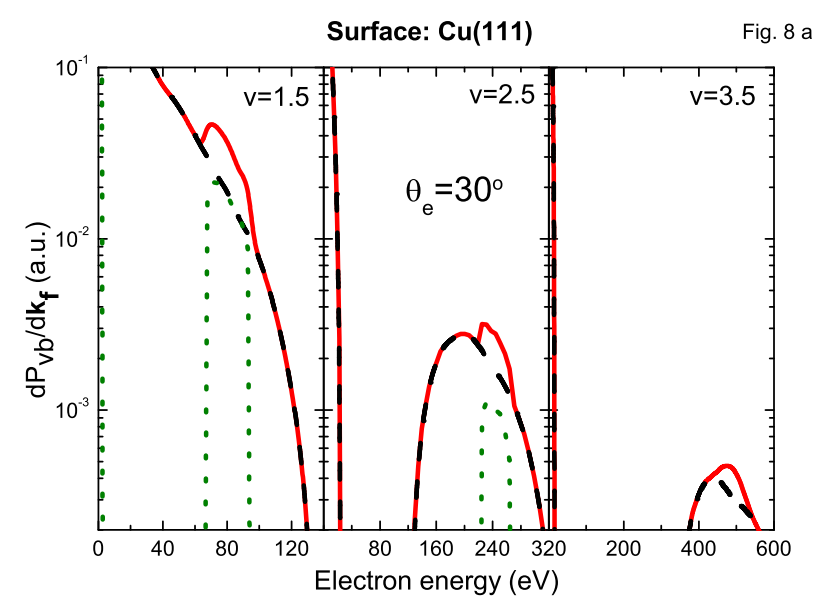

Fig. 8 b

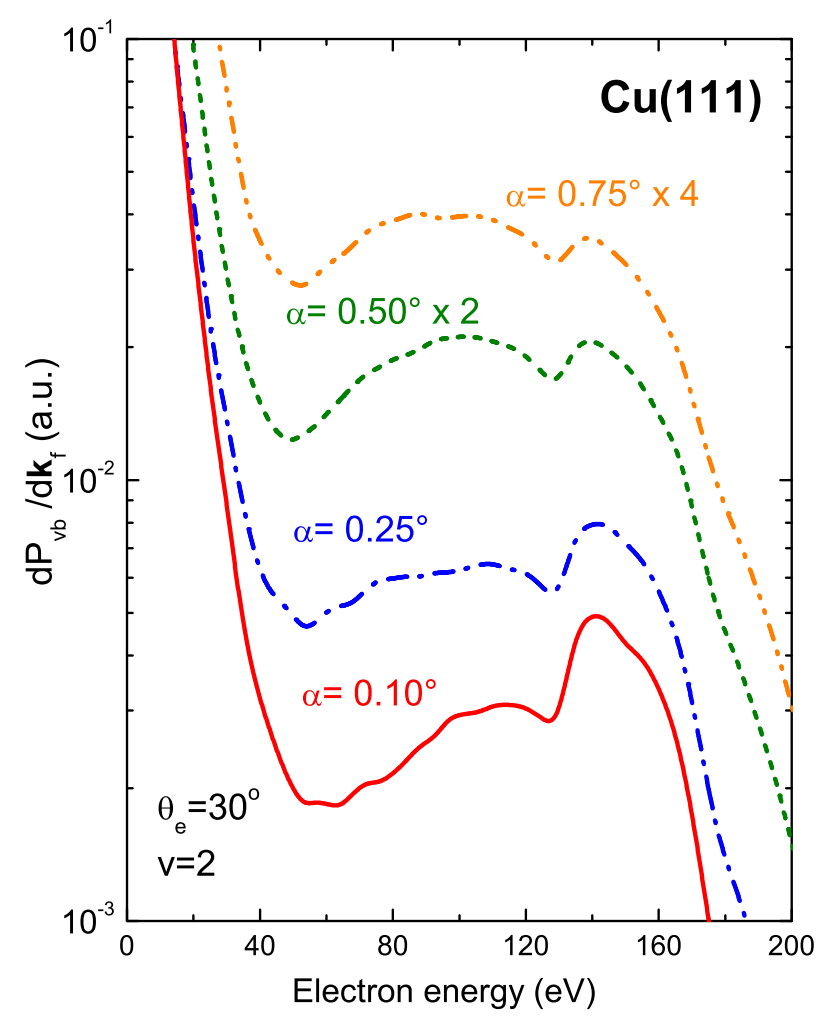

FIG. 8: (Color online) Influence of the incidence conditions on BSB electron emission probabilities from the valence band of $\mathrm{Cu}(111)$. The electron ejection angle is: $\theta_{e}=30^{\circ}$. (a) Different impact velocities, keeping the incidence angle, $\alpha=0.5^{\circ}$, as a constant. (b) Different incidence angles, keeping the impact velocity, $v=2$ a.u., as a constant. 